\title{
Direct photocoagulation to leakage points to treat chronic macular edema associated with branch retinal vein occlusion: a pilot study
}

This article was published in the following Dove Press journal:

Clinical Ophthalmology

7 October 2014

Number of times this article has been viewed

\section{Susumu Sakimoto \\ Motohiro Kamei \\ Hirokazu Sakaguchi \\ Mihoko Suzuki \\ Nagakazu Matsumura \\ Kentaro Nishida \\ Kohji Nishida}

Department of Ophthalmology, Osaka University Graduate School of Medicine, Suita, Osaka, Japan
Correspondence: Motohiro Kamei Department of Ophthalmology, Osaka University Graduate School of Medicine, E7, 2-2 Yamada-oka, Suita-shi, Osaka 565-087I, Japan

$\mathrm{Tel}+8 \mathrm{I} 668793455$

Fax +81668793458

Email mkamei@ophthal.med.osaka-u.ac.jp
Summary statement: Direct photocoagulation reduces the central foveal thickness (CFT) in cases with chronic branch retinal vein occlusion (BRVO) of longer than 12 months duration. Photocoagulation might be effective for chronic macular edema due to branch retinal vein occlusion.

Background: The aim was to investigate the effect of direct photocoagulation for treating chronic macular edema associated with BRVO.

Methods: This study was a noncomparative, pilot interventional case series. We examined the CFT and best-corrected visual acuity over 6 months in patients with BRVO treated with direct photocoagulation.

Results: Sixteen eyes of 16 patients had been treated with direct photocoagulation (mean follow-up period, 20.5 months). The mean CFT decreased significantly $(P<0.001)$ between the baseline $(465 \mu \mathrm{m})$ and the final visit $(304 \mu \mathrm{m})$. The mean (logarithm of the minimum angle of resolution equivalent) best-corrected visual acuity at the baseline was 0.39 and improved significantly $(P<0.001)$ to 0.20 at the final visit.

Conclusion: Direct photocoagulation to leakage points is beneficial for treating chronic macular edema associated with chronic BRVO of longer than 12 months duration.

Keywords: branch retinal vein occlusion, macular edema, optical coherence tomography, photocoagulation, VEGF

\section{Introduction}

Anti-vascular endothelial growth factor (VEGF) therapy using ranibizumab (Lucentis, Genentech, Inc., South San Francisco, CA, USA), an antibody fragment that specifically binds all isoforms of VEGF, has contributed substantially to the treatment of macular edema due to branch retinal vein occlusion (BRVO). The BRAnch Retinal Vein Occlusion: Evaluation of Efficacy and Safety [BRAVO]) study ${ }^{1}$ and An Extension Study to Evaluate the Safety and Tolerability of Ranibizumab in Subjects With Choroidal Neovascularization Secondary to AMD or Macular Edema Secondary to RVO (HORIZON) ${ }^{2}$ study showed that intraocular injection of ranibizumab for BRVO improved and maintained the best-corrected visual acuity (BCVA) for 2 years. However, the HORIZON study investigators reported that some patients required frequent injections during the 2 years of the study. The development of a new therapy for these patients remains to be elucidated. ${ }^{2}$

Aside from VEGF, the etiology of macular edema due to BRVO was assumed to be thrombosis of the branch retinal vein resulting in engorgement of the veins. ${ }^{3}$ The engorgement is followed by development of hemorrhages, edema, and retinal nonperfusion due to elevated intraluminal pressure. ${ }^{4}$ Several histopathological studies 
using trypsin digestion for specimen from patients with BRVO showed that some capillaries in the affected areas also became dilated, tortuous, and beaded. The investigators also reported that the vessels were usually characterized by acellularity of the capillary bed with rare pericytes. ${ }^{5,6}$ Recent basic research using a gene targeting system revealed a direct role of pericytes in barrier function and permeability control at the vessels in the central nervous system. ${ }^{7}$ To support this, fluorescein angiography (FA) findings indicated that those dilated, tortuous, and beaded abnormal vessels also included microaneurysms that were leaky and often contributed to macular edema. ${ }^{8,9}$ There is a possibility that certain rare leaky pericytes are refractory to anti-VEGF therapy because VEGF signaling generally is involved in endothelial cells. ${ }^{10}$

The Branch Vein Occlusion Study Group, the largest randomized prospective trial on this topic, reported the efficacy of grid-pattern laser photocoagulation for treating macular edema due to BRVO. ${ }^{11}$ The effectiveness of grid-pattern laser photocoagulation is thought to result from changes in the biochemical processes within the retinal pigment epithelium (RPE) or hypoxia in the neural retina. ${ }^{12,13}$ Meanwhile, in diabetic retinopathy, although the specific mechanism is unknown, the Early Treatment Diabetic Retinopathy Study found that focal laser photocoagulation, both grid and direct, reduces visual loss from diabetic macular edema. ${ }^{14}$ Among the findings in Early Treatment Diabetic Retinopathy Study, the potency of direct photocoagulation might result partly from closure of the microaneurysms. Actually, the mechanisms of BRVO and diabetic retinopathy differ; however, we hypothesized that direct photocoagulation could obstruct dilated vessels and microaneurysms in BRVO. In this pilot study, we explored the efficacy of direct photocoagulation for treating chronic macular edema in BRVO present for at least 12 months.

\section{Methods}

\section{Patient recruitment}

We conducted a noncomparative, nonrandomized pilot interventional study at the Department of Ophthalmology of Osaka University Hospital. The study followed the tenets of the Declaration of Helsinki. All patients provided written informed consent. Patients with BRVO were recruited. Eyes were included if they had macular edema that involved the fovea as diagnosed on slit-lamp biomicroscopy and a central foveal thickness (CFT) of $250 \mathrm{~mm}$ or greater measured by spectral domain optical coherence tomography (SD-OCT) (Cirrus HD-OCT; Carl Zeiss Meditec AG, Jena, Germany) and FA using a fundus camera (Topcon TRC50LX; Topcon,
Tokyo, Japan) or confocal scanning laser ophthalmoscope (Heidelberg Retina Angiograph; Heidelberg Engineering, Heidelberg, Germany). The study group included patients with BRVO who underwent direct photocoagulation 12 months after disease onset between January 2010 and August 2012. All patients had been referred to our institution within 3 months after visual loss. The exclusion criteria included a follow-up period shorter than 6 months after direct photocoagulation; the presence of central or hemicentral retinal vein occlusion; association with hemorrhage that was detected within the areas of treatment; severe cataract and other retinal disorders such as epiretinal membranes, macular holes, diabetic retinopathy; or a history of retinal photocoagulation. We also excluded patients who underwent direct photocoagulation at least 3 months after treatment that included a drug injection, and those who underwent grid photocoagulation during the study period.

\section{Treatment intervention}

FA-guided direct photocoagulation was applied to areas of dilated and leaking capillaries, and highly permeable microaneurysms within vascular arcade. The field of view was digitally magnified at angles of $15^{\circ}$ in standard resolution. In the $15 \times 15$-degree scan, fine perifoveal capillaries are well delineated. ${ }^{15}$ Retinal vascular changes associated with BRVO were seen in the early to middle phase FA images. ${ }^{15}$ Direct photocoagulation was performed with the following parameters: argon laser wavelength, $532 \mathrm{~nm}$; spot size, $50 \mu \mathrm{m}$; laser power, $100-150 \mathrm{~mW}$; and pulse duration, $50 \mathrm{~ms}$ using a Goldmann three-mirror contact lens. The beam was aimed at the dilated capillaries or microaneurysms, which were present in the inner retinal layer. Care was taken to avoid coagulation of the RPE layer and foveal avascular area.

\section{Outcome measures}

The primary outcome measures were the changes in the mean CFT and average thickness on OCT; the secondary outcome measure was the BCVA. At the baseline, which was just before the direct photocoagulation, the BCVA was measured using a decimal chart and calculated as the logarithm of the minimum angle of resolution. The CFT was defined as the distance between the inner retinal surface and the inner border of the RPE measured using SD-OCT Stratus 4 software (Carl Zeiss Meditec AG) as the mean thickness at the point of intersection of six radial scans, acquired using the Fast Macular Thickness Map protocol; the value was generated automatically by the OCT instrument using the retinal map analysis function. 


\section{Data analysis}

Statistical analysis was performed using JMP software version 8.0 (SAS Institute Inc., Cary, NC, USA). Comparisons of paired data, eg, the baseline and postinjection BCVA and CFT values, were performed using the paired $t$-test (for parametric data) or the Wilcoxon signed rank tests (for nonparametric data). $P<0.05$ was considered statistically significant.

\section{Results}

Sixteen eyes of 16 patients (seven men, nine women; mean age, 72.0 \pm 6.3 years; range, 60-87) were recruited for the current study. Table 1 shows the patient demographics and the baseline characteristics. The number of eyes with cotton wool spots was four eyes, and the number of eyes with retinal nonperfusion was five eyes. The mean period between the first visit and the first application of direct photocoagulation was $20.9 \pm 9.9$ months (range, 12-45 months). The mean follow-up period from the first direct photocoagulation was $20.3 \pm 8.0$ months (range, 9-32 months). The mean number of applications of direct photocoagulation was $1.7 \pm 0.9$ (range, $0-3)$. An additional procedure after direct photocoagulation, ie, sub-Tenon injection of triamcinolone and intravitreal injection of bevacizumab, was performed to treat recurrent macular edema in four eyes $(25.0 \%)$ and one eye $(6.3 \%)$. In terms of a drug injection before direct photocoagulation, sub-Tenon injection of triamcinolone was performed in ten $(62.5 \%)$ eyes, intravitreal injection of triamcinolone was performed in two (12.5\%) eyes, and intravitreal injection of bevacizumab was performed in eight $(50.0 \%)$ eyes. The mean period between these additional procedures and the first application of direct photocoagulation was $5.69 \pm 2.5$ months (range, 3-10 months). Scatter laser photocoagulation was performed to treat an area of nonperfusion in one $(6.3 \%)$ eye.

The mean baseline CFT was $465.0 \pm 107.6 \mu \mathrm{m}$ (range, 317-746 $\mu \mathrm{m})$. The mean CFTs 3 and 6 months after laser treatment were $355.3 \pm 91.3 \mu \mathrm{m}$ and $334.3 \pm 68.8 \mu \mathrm{m}$, respectively, which differed significantly $(P<0.01$ for both comparisons) from the baseline. The mean final CFT was $303.9 \pm 51.7 \mu \mathrm{m}$, which also differed significantly $(P<0.001)$ from the baseline (Figure 1).

The mean baseline BCVA was $0.39 \pm 0.28$ (range, $0.05-1.00$ ). The mean BCVAs 3 and 6 months after laser treatment were $0.31 \pm 0.23$ (range, -0.08 to 0.70 ) and $0.24 \pm 0.22$ (range, -0.08 to 0.70$)$. Each differed significantly $(P=0.016$ and $P=0.0024$, respectively) from the baseline. The mean final BCVA was $0.20 \pm 0.23$ (range, -0.08 to 0.70 ), which differed significantly $(P=0.0011)$ from the baseline (Table 2).

\section{Case}

A 68-year-old woman was referred to our hospital with blurred vision in the left eye. After an ophthalmic examination, she was diagnosed with BRVO in her left eye with a VA of 120/200 and was treated with a sub-Tenon injection of triamcinolone. An intravitreal injection of bevacizumab (Avastin, Genentech, Inc.) for recurrent macular edema was administered 5 months after the triamcinolone injection and was repeated due to a second recurrence. Direct photocoagulation was performed 12 months after the initial visit. OCT showed a substantial decrease in the macular edema in the central fovea from 552 to $275 \mu \mathrm{m} 3$ months after direct photocoagulation compared to the baseline. FA showed fewer microaneurysms and less leakage after direct

Table I Direct photocoagulation to treat macular edema associated with BRVO: patient demographics

\begin{tabular}{ll}
\hline Sex, number (\%) & $7(43.8 \%)$ \\
Men & $9(56.3 \%)$ \\
Women & $72.0 \pm 6.3(60-87)$ \\
Average age (years \pm standard deviation) (range) & I0 (47.6\%) \\
Site of vascular occlusion (\%) & II (52.4\%) \\
$\quad$ Lower quartile & $20.3 \pm 8.0$ months \\
Upper quartile & $($ range, 9-32) \\
Mean follow-up period from the first direct PC & $0.3 \pm 0.6($ (range, 0-2) \\
(months \pm standard deviation) (range) & $4(25.0 \%)$ \\
Mean number of applications of direct PC & I (6.3\%) \\
(number \pm standard deviation) (range) & I (6.3\%) \\
Number of eyes with additional procedure after direct photocoagulation (\%) & \\
Sub-Tenon injection of triamcinolone &
\end{tabular}

Abbreviations: BRVO, branch retinal vein occlusion; PC, photocoagulation. 
A

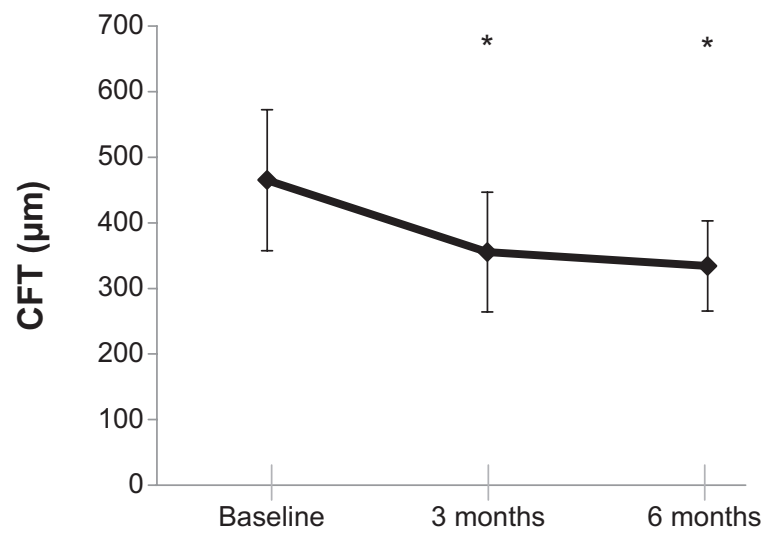

B

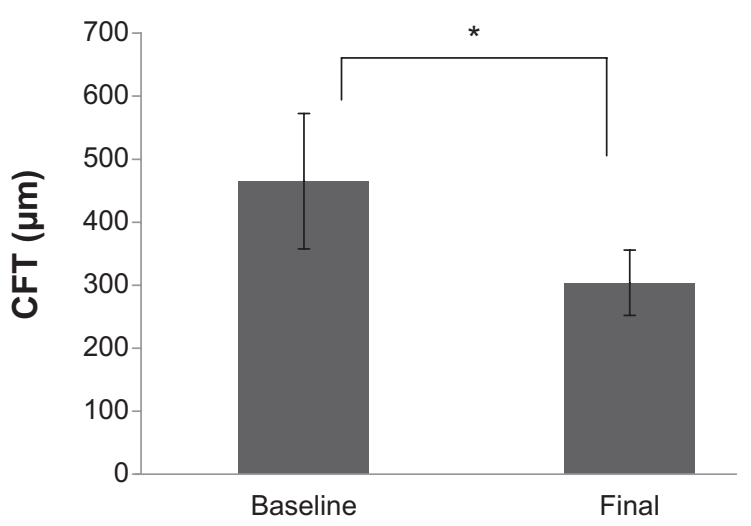

Figure I Reduction in the central foveal thickness (CFT) after direct photocoagulation.

Notes: (A) The line graph shows the changes in the CFT 3 months and 6 months after direct photocoagulation. $* P<0.0$ I by one-way analysis of variance and the Tukey's test. (B) The bar graph shows a comparison of the baseline and final CFTs. $* P<0.01$, by the paired $t$-test.

photocoagulation (Figure 2). The BCVA improved from $40 / 200$ at the baseline to $60 / 200$ at the final visit.

\section{Discussion}

In the current study, direct photocoagulation effectively treated chronic macular edema associated with BRVO that was present for longer than 12 months. The CFT decreased significantly after direct photocoagulation, and the effect was maintained for at least 6 months. Moreover, the visual outcomes after direct photocoagulation improved significantly compared to the baseline.

Although previously randomized clinical trials have reported the efficacy of ranibizumab for BRVO, ${ }^{1,2}$ several case series have reported frequent recurrences of macular edema even after multiple injections of the drug. ${ }^{16-18}$ In addition, the appropriate duration of anti-VEGF therapy remains to be elucidated. Photocoagulation therapy, in contrast to drug therapy, might be expected to have a long-term effect; however, the detailed mechanism of grid photocoagulation is not fully understood. In addition, the effect of combined therapy including anti-VEGF therapy and laser has not been clarified.

Table 2 Visual outcomes after direct photocoagulation for chronic macular edema associated with BRVO

\begin{tabular}{llll}
\hline & Mean BCVA & SD & P-value \\
\hline Baseline & 0.39 & 0.28 & \\
3 months & 0.31 & 0.23 & $0.016^{*}$ \\
6 months & 0.24 & 0.22 & $0.0024^{* *}$ \\
Final & 0.20 & 0.23 & $0.0011^{* *}$ \\
\hline
\end{tabular}

Notes: $* P<0.05, * * P<0.01$, by the paired $t$-test compared with the baseline.

Abbreviations: BRVO, branch retinal vein occlusion; BCVA, best-corrected visual acuity; SD, standard deviation.
Frangieh et $\mathrm{al}^{6}$ using $\mathrm{FA}$, reported that venules and capillaries had irregular borders and that tortuous and capillary microaneurysms were present in clusters around a vein in patients with BRVO. We postulated that these abnormal vessels were acellular, rare pericytes that were refractive to anti-VEGF therapy. Because VEGFR-2, which is activated by VEGF-A, is specifically expressed on the vascular endothelial cells, ${ }^{8}$ a therapy targeting VEGF signaling had little effect on the leaky vessels without pericytes. Taken together with the treatment for the microaneurysms in diabetic macular edema, we performed direct photocoagulation to close the abnormal vessels containing dilated, tortuous capillaries and microaneurysms.

The effect of direct photocoagulation was relevant to the significant reduction of macular edema in the current pilot study; however, a fundamental question arose about whether we actually transmitted laser energy directly to the vessel or microaneurysms. In addition, the mechanism of the collapse of the microvessels is unknown. We selected the $577 \mathrm{~nm}$ yellow laser wavelength because the objective contained a large quantity of hemoglobin. Elevated temperature of the blood flow induces changes in the conformation of various proteins in the vessel walls, which denature at characteristic temperatures specific to the protein species. Although coagulation of blood vessels requires more energy than other stationary tissue due to cooling of the blood flow, we set a short duration time for the laser pulse to efficiently generate more heat in the blood flow. We also confirmed coagulation of the blood vessels based on the appearance of white scars that corresponded to the burned dilated capillaries or microaneurysms. FA after direct photocoagulation showed decreased leakage; however, complete obstruction of vessels 

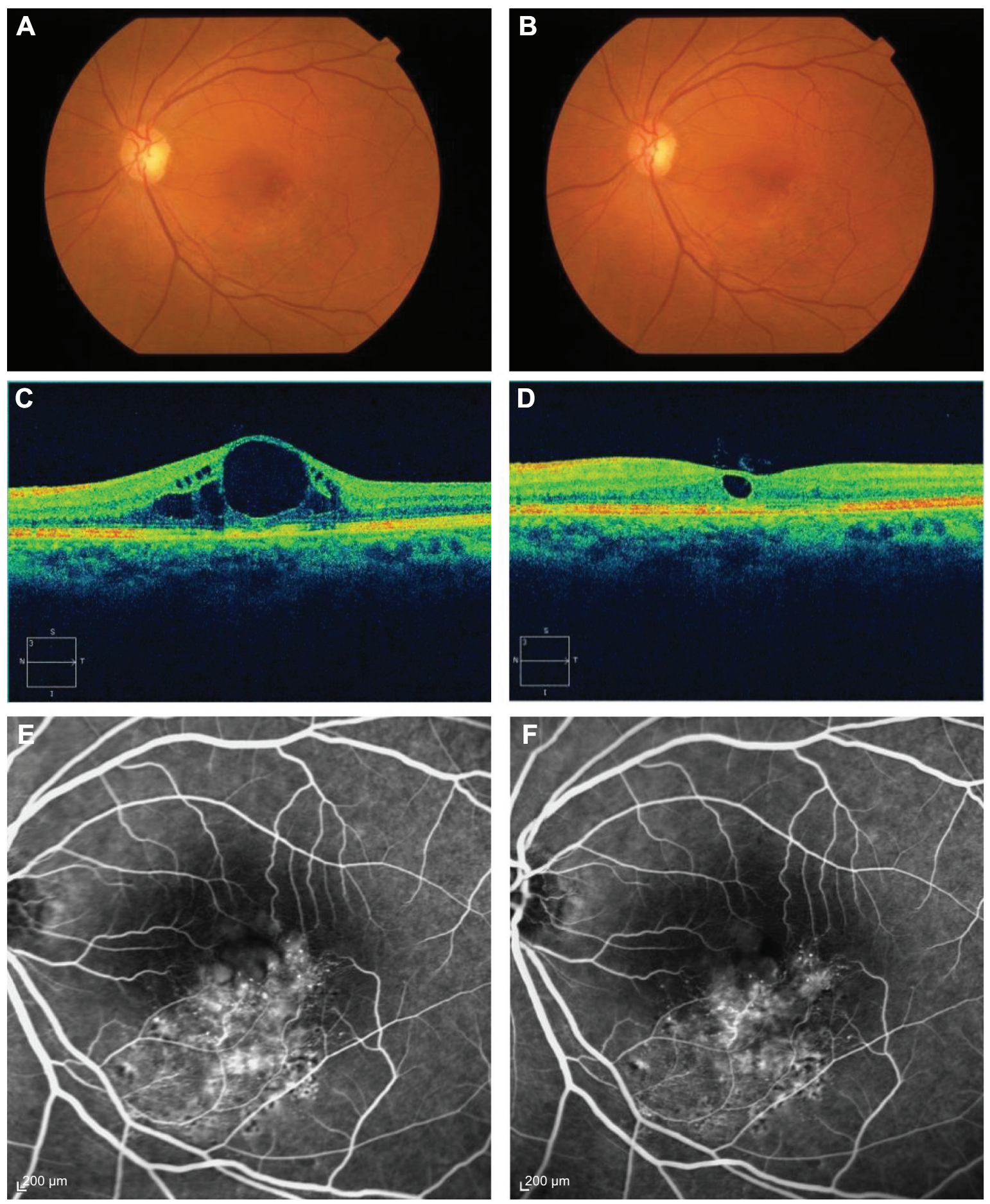

Figure 2 Images of the left eye of a 68-year-old woman with branch retinal vein occlusion (BRVO).

Notes: A fundus photograph (top), optical coherence tomography (OCT) image (middle), and fluorescein angiography (FA) image (bottom) were obtained at baseline (A, C, E) and 3 months (B, D, F) after direct photocoagulation. (D) OCT shows decreased macular edema after direct photocoagulation compared to the baseline (C). FA shows decreased microaneurysms and leakage after direct photocoagulation. The best-corrected visual acuity (BCVA) improved from 40/200 to 60/200.

was rare (Figure 2). In the present study, we needed several sessions in some patients.

The detailed mechanisms of the effect of direct photocoagulation on leaky vessels remain to be determined. A larger sample size, a comparative group, a controlled treatment schedule, and a longer follow-up period are required for a study with appropriate analysis to detect the efficacy of direct photocoagulation. Therefore, the current study does not have the structure or power to determine whether direct photocoagulation is more effective than a standard treatment 
such as an intravitreal injection of an anti-VEGF antibody. To demonstrate this, we would need a large-scale randomized study with contemporary controls with at least 2 years of follow-up. Our intention was to perform this pilot study to determine if laser treatment can reduce chronic macular edema, which is sufficient to justify further study. However, regarding photocoagulation, there might be a learning curve associated with this procedure. Because the targets and the size of the laser beam were small, involuntary ocular movement could contribute to an increased level of difficulty.

In conclusion, in the current small noncomparative pilot study, we observed the efficacy of direct photocoagulation for treating macular edema, even 12 months after the disease onset. Direct photocoagulation applied to treat chronic macular edema might result in sustained decreases of the CFT up to 1.5 years after treatment. Furthermore, we found a relatively small but significant improvement in BCVA after laser treatment.

\section{Disclosure}

The authors have no conflicts of interest in this work.

\section{References}

1. Brown DM, Campochiaro PA, Bhisitkul RB, et al. Sustained benefits from ranibizumab for macular edema following branch retinal vein occlusion: 12-month outcomes of a phase III study. Ophthalmology. 2011;118: 1594-1602.

2. Heier JS, Campochiaro PA, Yau L, et al. Ranibizumab for macular edema due to retinal vein occlusions: long-term follow-up in the HORIZON trial. Ophthalmology. 2012;119:802-809.

3. Phillips S, Fekrat S, Finkelstein D. Branch retinal vein occlusion. In: Ryan SJ, editor. Retina. 4th ed. St. Louis: Mosby; 2006:1349-1354.

4. Campochiaro PA, Bhisitkul RB, Shapiro H, Rubio RG. Vascular endothelial growth factor promotes progressive retinal nonperfusion in patients with retinal vein occlusion. Ophthalmology. 2013;120:795-802.
5. Kornzweig AL, Eliasoph I, Feldstein M. Occlusive disease of retinal vasculature. A clinicopathologic study. Arch Ophthalmol. 1964;71: 542-551.

6. Frangieh GT, Green WR, Barraquer-Somers E, Finkelstein D. Histopathologic study of nine branch retinal vein occlusions. Arch Ophthalmol. 1982;100:1132-1140.

7. Armulik A, Genové G, Mäe M, et al. Pericytes regulate the blood-brain barrier. Nature. 2010;468:557-561.

8. Finkelstein D. Ischemic macular edema: recognition and favorable natural history in branch vein occlusion. Arch Ophthalmol. 1992;110: $1427-1434$.

9. Sakimoto S, Kamei M, Suzuki M, et al. Relationship between grades of macular perfusion and foveal thickness in branch retinal vein occlusion. Clin Ophthalmol. 2013;7:39-45.

10. Carmeliet $P$, Jain RK. Molecular mechanisms and clinical applications of angiogenesis. Nature. 2011;473:298-307.

11. Branch Vein Occlusion Study Group. Argon laser photocoagulation for macular edema in branch vein occlusion. Am J Ophthalmol. 1984; 98:271-282.

12. Ogata N, Tombran-Tink J, Jo N, Mrazek D, Matsumura M. Upregulation of pigment epithelium-derived factor after laser photocoagulation. Am J Ophthalmol. 2001;132:427-429.

13. Xiao M, McLeod D, Cranley J, Williams G, Boulton M. Growth factor staining patterns in the pig retina following retinal laser photocoagulation. Br J Ophthalmol. 1999;83:728-736.

14. Early Treatment Diabetic Retinopathy Study Research Group. Photocoagulation for diabetic macular edema. Early Treatment Diabetic Retinopathy Study report number 1. Arch Ophthalmol. 1985;103: 1796-1806.

15. Jorzik JJ, Bindewald A, Dithmar S, Holz FG. Digital simultaneous fluorescein and indocyanine green angiography, autofluorescence, and red-free imaging with a solid-state laser-based confocal scanning laser ophthalmoscope. Retina. 2005;25:405-416.

16. Kreutzer TC, Alge CS, Wolf AH, et al. Intravitreal bevacizumab for the treatment of macular oedema secondary to branch retinal vein occlusion. Br J Ophthalmol. 2008;92:351-355.

17. Rabena MD, Pieramici DJ, Castellarin AA, Nasir MA, Avery RL. Intravitreal bevacizumab (Avastin) in the treatment of macular edema secondary to branch retinal vein occlusion. Retina. 2007;27:419-425.

18. Hanada N, Iijima H, Sakurada Y, Imasawa M. Recurrence of macular edema associated with branch retinal vein occlusion after intravitreal bevacizumab. Jpn J Ophthalmol. 2012;56:165-174.
Clinical Ophthalmology

\section{Publish your work in this journal}

Clinical Ophthalmology is an international, peer-reviewed journal covering all subspecialties within ophthalmology. Key topics include: Optometry; Visual science; Pharmacology and drug therapy in eye diseases; Basic Sciences; Primary and Secondary eye care; Patient Safety and Quality of Care Improvements. This journal is indexed on Submit your manuscript here: http://www.dovepress.com/clinical-ophthalmology-journal

\section{Dovepress}

PubMed Central and CAS, and is the official journal of The Society of Clinical Ophthalmology (SCO). The manuscript management system is completely online and includes a very quick and fair peer-review system, which is all easy to use. Visit http://www.dovepress.com/ testimonials.php to read real quotes from published authors. 